\title{
A Case of Tyrosine Kinase Inhibitor- Induced Bone Marrow Aplasia That Was Successfully Treated with Allogeneic Hematopoietic Stem Cell Transplantation
}

\author{
Kimimori Kamijo ${ }^{a}$ Yoshimitsu Shimomura $^{a}$ Daisuke Yamashita ${ }^{b}$ \\ Takayuki Ishikawa ${ }^{a}$ \\ aDepartment of Hematology, Kobe City Hospital Organization Kobe City Medical Center \\ General Hospital, Kobe, Japan; bepartment of Pathology, Kobe City Hospital Organization \\ Kobe City Medical Center General Hospital, Kobe, Japan
}

\section{Keywords}

Tyrosine kinase inhibitors-induced bone marrow aplasia · Chronic myeloid leukemia . Hematopoietic stem cell transplantation

\section{Abstract}

Here, we present a rare case of tyrosine kinase inhibitor (TKI)-induced bone marrow aplasia. A 58-year-old man presented with leukocytosis and was diagnosed with chronic myeloid leukemia. He was initially treated with imatinib for 6 years and abruptly discontinued treatment by himself. He was administered dasatinib 5 years after treatment interruption, and presented with pancytopenia 6 months after dasatinib initiation. Bone marrow biopsy revealed severe hypocellularity without blasts. Dasatinib was discontinued, and he recovered from pancytopenia 3 months later; however, $B C R-A B L 1$ was positive for almost all white blood cells in the peripheral blood. We retreated with ponatinib, but pancytopenia developed again. The clinical course indicated TKI-induced bone marrow aplasia. Therefore, ponatinib was discontinued and the patient received an allogeneic hematopoietic stem cell transplantation from a haploidentical daughter using post-transplant cyclophosphamide. He had a major molecular response and had normal complete blood counts and bone marrow 1 year after transplantation. 


\section{Introduction}

Chronic myeloid leukemia (CML) is a myeloproliferative neoplasm typically associated with the Philadelphia chromosome, which is derived from a reciprocal translocation between chromosomes 9 and 22, resulting in oncogenic BCR-ABL1 gene fusion $[1,2]$. The prognosis of CML has improved with the development of small molecule tyrosine kinase inhibitors (TKIs), which offer the prospect of long-term disease management with a simple oral therapy [2]. As a result, survival rates in patients with CML are approaching that of the general population [3]; however, nearly all patients who receive TKIs experience 1 or more adverse events [4]. Hematological adverse events are common, occurring in up to $70 \%$ of patients, but most of them are reversible by treatment cessation or dose reduction [5]. For example, a previous phase 3 study that compared the safety of 2 TKIs, dasatinib, and imatinib, showed that about a quarter of the patients developed grade 3-4 cytopenias; a few patients discontinued treatment due to this side effect [6]. TKI-induced bone marrow aplasia, which develops under persistent cytopenia, is a particularly rare adverse event and is only reported in select case reports. This adverse event may make it difficult to continue TKIs, which decreases the therapeutic efficacy of the treatment. The exact mechanism is still unknown, and there are no appropriate treatment strategies to mitigate this effect $[7,8]$. Herein, we report the case of a patient who developed TKI-induced bone marrow aplasia and was successfully treated with allogeneic hematopoietic stem cell transplantation (allo-HSCT).

\section{Case Report/Case Presentation}

This case report did not require review by the institutional review board of our hospital. Written informed consent for treatment and publication was obtained from the patient.

A 58-year-old man with no comorbidities presented with leukocytosis, which was revealed during a health check-up. The complete blood count (CBC) showed a white blood cell (WBC) count of $46.2 \times 10^{3} / \mu \mathrm{L}$, a hemoglobin (Hb) level of $13.8 \mathrm{~g} / \mathrm{dL}$, and a platelet (Plt) count of $39.2 \times 10^{4} / \mu \mathrm{L}$. Bone marrow examination showed hypercellular with myeloid hyperplasia with $0.8 \%$ blast cells. Chromosomal examination showed 46 XY, t (9; 22) (q34; q11) and fluorescence in situ hybridization (FISH) analysis showed positive BCR-ABL1 fusion gene in $99.6 \%$ of the cells. He was diagnosed with chronic phase CML. He was initially treated with imatinib and abruptly discontinued treatment by himself 6 years after treatment initiation.

He returned to our hospital 5 years after the cessation of treatment. He had leukocytosis with a WBC count of $233.2 \times 10^{3} / \mu \mathrm{L}$ and $18 \%$ of blasts in peripheral blood. He was diagnosed with accelerated phase CML and was retreated with dasatinib. The BCR-ABL1 on the International Scale $\left(B C R-A B L 1^{1 \mathrm{~S}}\right)$ after 3 months of treatment with dasatinib (100 $\mathrm{mg}$ ) was above $10 \%$, but gradually improved from $97.39 \%$ to $36.62 \%$. We increased dasatinib from 100 to $150 \mathrm{mg}$ and the $B C R-A B L 1^{\mathrm{IS}}$ was 20.51 after 5 months of treatment.

He was admitted to our hospital because of general fatigue and melena 6 months after initiation of dasatinib when he was 69 years old. The CBC showed an abnormally decreased WBC count of $1.2 \times 10^{3} / \mu \mathrm{L}$, Hb level of $2.5 \mathrm{~g} / \mathrm{dL}$, and Plt count of $0.7 \times 10^{4} / \mu \mathrm{L}$. Serologic testing for human immunodeficiency virus, hepatitis A, hepatitis B, hepatitis C, and parvovirus (IgM) were negative. Bone marrow biopsy revealed severe hypocellularity $(<5 \%$ of normal cellularity; shown in Fig. 1a) without blasts. Cytogenetic analysis revealed 46 XY, t (9; 22) (q34; q11) (1/2). Dasatinib was discontinued because we suspected dasatinib as a cause of bone marrow aplasia.

Three months later, $\mathrm{CBC}$ was recovered with a WBC count of $5.2 \times 10^{3} / \mu \mathrm{L}$, Hb level of 9.8 $\mathrm{g} / \mathrm{dL}$, and Plt count of $13.3 \times 10^{4} / \mu \mathrm{L}$; however, FISH analysis for $B C R-A B L 1$ in peripheral blood 


\section{Case Reports in Oncology}

\begin{tabular}{l|l}
\hline Case Rep Oncol 2021;14:1139-1143 \\
\hline DOI: 10.1159/000517442 & $\begin{array}{l}\text { @ 2021 The Author(s). Published by S. Karger AG, Basel } \\
\text { www.karger.com/cro }\end{array}$ \\
\hline
\end{tabular}

Kamijo et al.: Stem Cell Treatment of Tyrosine Kinase Inhibitor-Induced Bone Marrow Aplasia
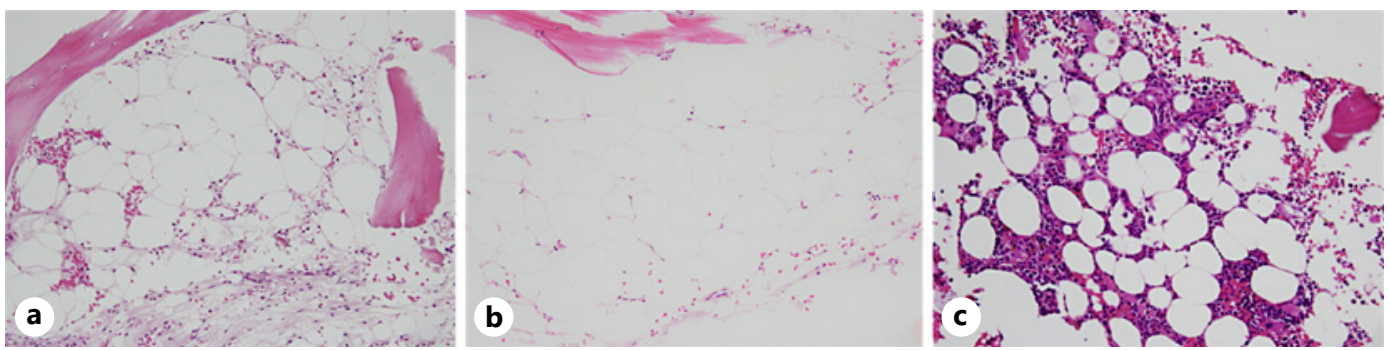

Fig. 1. Imaging results. Hematoxylin and eosin staining of bone marrow biopsy following pancytopenia, showing hypoplasia (bone marrow cellularity $\leqq 5 \%)(\times 20)(a)$; bone marrow biopsy before transplantation $(\times 20)($ b); bone marrow biopsy 1 year after transplantation $(\times 20)(\mathbf{c})$.

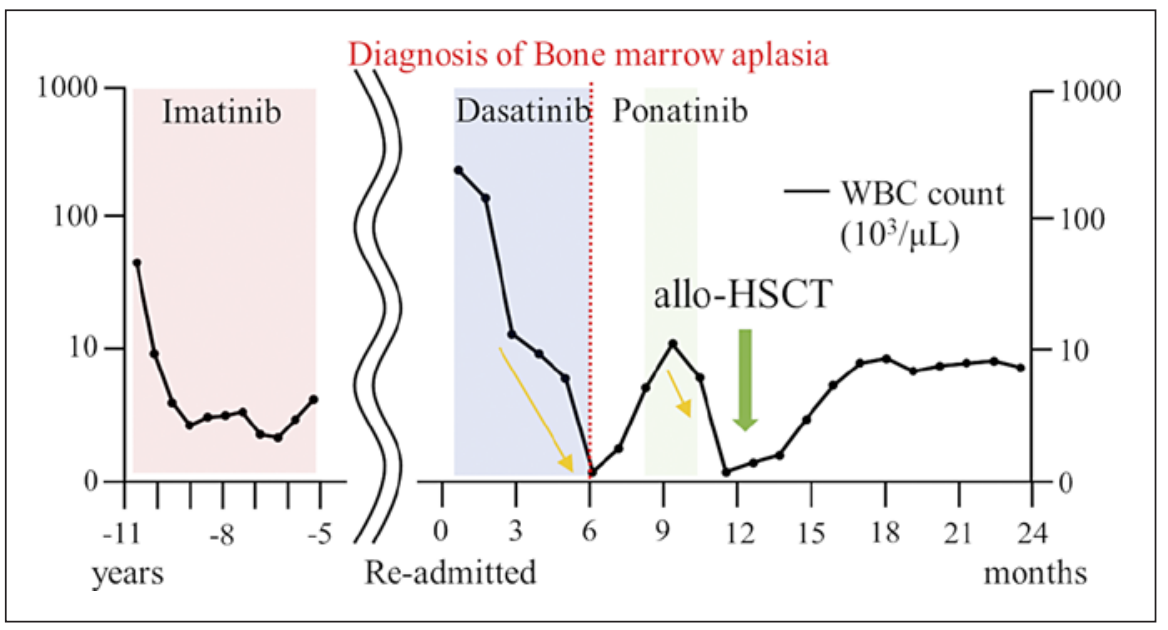

Fig. 2. Clinical course. Allo-HSCT, allogeneic hematopoietic stem cell transplantation; WBC, white blood cell.

was positive for $95 \%$ of cells. We administered ponatinib, but the bone marrow aplasia developed again (shown in Fig. 1b). Cytogenetic analysis of the bone marrow revealed $46 \mathrm{XY}$, t $(9 ; 22)$ (q34; q11) (20/20). The clinical course indicated that he was diagnosed with TKIinduced bone marrow aplasia and any TKIs would cause bone marrow aplasia. Therefore, he received allo-HSCT from a haploidentical daughter using post-transplant cyclophosphamide. A reduced-intensity conditioning regimen consisting of fludarabine $\left(50 \mathrm{mg} / \mathrm{m}^{2}\right.$ daily for 6 days), busulfan ( $3.2 \mathrm{mg} / \mathrm{kg}$ daily for 2 days), and total body irradiation (4 Gy) was used.

He achieved successful neutrophil engraftment on day +15 . He had a major molecular response and had normal CBCs and bone marrow 1 year after transplantation (shown in Fig. 1c). The clinical course is shown in Figure 2.

\section{Discussion/Conclusion}

In our case, the patient developed severe pancytopenia during TKI treatment. Bone marrow examination showed bone marrow aplasia with cellularity of $<5 \%$. Severe pancytopenia improved after discontinuation of dasatinib but occurred again after initiation of ponatinib. He received allo-HSCT with residual disease and successfully improved bone marrow aplasia, achieving a major molecular response in CML. This case report suggests that alloHSCT is an effective option for TKI-induced bone marrow aplasia. 
The mechanism of TKI-induced bone marrow aplasia is still unknown. In broad terms, there are 2 possible mechanisms. First, TKIs can directly interfere with normal hematopoiesis. TKIs inhibit not only $B C R / A B L$ kinase activity in CML cells but also other tyrosine kinases, such as c-KIT and platelet-derived growth factor receptors, which are involved in hematopoiesis $[9,10]$. Of note, imatinib has a known inhibitory effect on normal CD34 positive progenitor cells $[11,12]$. The second option is that normal hematopoiesis is suppressed by CML cells. TKIs can rapidly and effectively clear CML cells; therefore, if the CML clones fill the bone marrow, TKIs create an empty marrow with few normal hematopoietic cells, which results in insufficient hematopoiesis [7]. Other possibilities, including autoimmune response (aplastic anemia) [12] and myelofibrosis [13], have also been reported. The first and second mechanisms may be involved, but it is unclear to what extent.

The appropriate treatment strategies for TKI-induced bone marrow aplasia are unclear because of its rarity. In almost all previously reported cases including our case, patients discontinued TKIs and improved pancytopenia at least temporarily, although some cases persisted for approximately several months after discontinuation of TKIs [8, 11]. Conversely, subsequent treatments and their effectiveness differed among cases. Some cases revealed that the re-administration of TKIs successfully treated patients without recurrent bone marrow aplasia. For example, a 53-year-old CML patient who developed bone marrow aplasia during treatment with nilotinib was retreated with dasatinib without the development of a hematological adverse event [7]. The patient had no CML cells at the time of the TKI-induced bone marrow aplasia diagnosis, discontinued the problematic TKIs, and changed to more suitable TKIs. The treatment strategy resulted in the recovery of blood cells and good control of CML [7]. On the other hand, TKI retreatment induced TKI-induced bone marrow aplasia in some reports, including our case [14]. Patients who had poorly managed CML at the time of diagnosis typically developed bone marrow aplasia again following retreatment with TKIs, which resulted in a fatal outcome due to pancytopenia [14]. Notably, some of the patients who received allo-HSCT, including our case, were rescued [8]. Therefore, we hypothesize that the treatment outcome is associated with the presence or absence of residual CML cells at the time of diagnosis of TKI-induced bone marrow aplasia. Furthermore, the treatment strategy may change according to the presence of the CML clone. That is, if CML cells remain at the onset of TKI-induced bone marrow aplasia, allo-HSCT is an option that will prevent fatal complications due to bone marrow aplasia.

In conclusion, we presented a rare case of TKI-induced bone marrow aplasia in a patient with CML. The precise mechanism of TKI-induced bone marrow aplasia is unknown. Our case suggests the effectiveness of allo-HSCT for CML patients with TKI-induced bone marrow aplasia.

\section{Acknowledgments}

The authors thank the medical and nursing staff at Kobe City Medical Centre General Hospital.

\section{Statement of Ethics}

This case report did not require review by the institutional review board of our hospital. Written informed consent for treatment and publication was obtained from the patient.

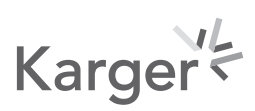




\section{Conflict of Interest Statement}

The authors have no conflicts of interest to declare.

\section{Funding Sources}

The authors have no funding to report.

\section{Author Contributions}

All authors have read and approved the manuscript. K.K. and Y.S. wrote the manuscript. K.K., D.Y., and T.I. contributed to the diagnosis and treatment. D.Y. performed the pathologic examination.

\section{References}

1 Schiffer CA. Bcr-abl tyrosine kinase inhibitors for chronic myelogenous leukemia. N Engl J Med. 2007;357(3): 258-65.

2 Innes AJ, Milojkovic D, Apperley JF. Allogeneic transplantation for CML in the TKI era: Striking the right balance. Nat Rev Clin Oncol. 2016;13(2):79-91.

3 Bower H, Björkholm M, Dickman PW, Höglund M, Lambert PC, Andersson TM. Life expectancy of patients with chronic myeloid leukemia approaches the life expectancy of the general population. J Clin Oncol. 2016;34(24): 2851-7.

4 Caldemeyer L, Dugan M, Edwards J, Akard L. Long-term side effects of tyrosine kinase inhibitors in chronic myeloid leukemia. Curr Hematol Malig Rep. 2016;11(2):71-9.

5 Steegmann JL, Baccarani M, Breccia M, Casado LF, García-Gutiérrez V, Hochhaus A, et al. European leukemianet recommendations for the management and avoidance of adverse events of treatment in chronic myeloid leukaemia. Leukemia. 2016;30(8):1648-71.

6 Kantarjian H, Shah NP, Hochhaus A, Cortes J, Shah S, Ayala M, et al. Dasatinib versus imatinib in newly diagnosed chronic-phase chronic myeloid leukemia. N Engl J Med. 2010;362(24):2260-70.

7 Estephan F, Rogers HJ, Visconte V, Tabarroki A, Ai J, Duong HK, et al. Spontaneous recovery of severe nilotinibinduced bone marrow aplasia and successful retreatment with dasatinib in a patient with chronic phase chronic myeloid leukemia. Leuk Lymphoma. 2015;56(3):811-3.

8 Cervetti G, Carulli G, Galimberti S, Azzarà A, Buda G, Orciuolo E, et al. Transitory marrow aplasia during imatinib therapy in a patient with chronic myeloid leukemia. Leuk Res. 2008;32(1):194-5.

9 Prodduturi P, Perry AM, Aoun P, Weisenburger DD, Akhtari M. Recurrent bone marrow aplasia secondary to nilotinib in a patient with chronic myeloid leukemia: a case report. J Oncol Pharm Pract. 2012;18(4):440-4.

10 Kennedy JA, Hobbs G. Tyrosine kinase inhibitors in the treatment of chronic-phase CML: strategies for frontline decision-making. Curr Hematol Malig Rep. 2018;13(3):202-11.

11 Chng WJ, Tan LH. Late-onset marrow aplasia due to imatinib in newly diagnosed chronic phase chronic myeloid leukaemia. Leuk Res. 2005;29(6):719-20.

12 LeMarbre G, Schinstock C, Hoyer R, Krook J, Tefferi A. Late onset aplastic anemia during treatment of chronic myeloid leukemia with imatinib mesylate. Leuk Res. 2007;31(3):414-5.

13 Song MK, Choi YJ, Seol YM, Shin HJ, Chung JS, Cho GJ, et al. Nilotinib-induced bone marrow aplasia. Eur J Haematol. 2009;83(2):161-2.

14 Ramdial JL, Aguirre LE, Ali RA, Swords R, Goodman M. Aplasia in chronic phase CML post-TKI therapy: a management dilemma. Case Rep Hematol. 2019;2019:4861673.

\section{Karger'א}

